\title{
Cranial Venous Outflow Obstruction Promotes Neuroinflammation And Edema Via ADAM17/solTNF-a/NF-kB Pathway In A Rat Subdural Hematoma Model
}

\section{Cheng Wang}

Fujian Medical University

\section{Liang Xian}

Fujian Medical University

\section{Shaorui Zheng}

Fujian Medical University

\section{Yi Fang}

Fujian Medical University

Kunzhe Lin

Fujian Medical University

\section{Zihuan Zeng}

Fujian Medical University

Jun Li

Fujian Medical University

Shousen Wang ( $\nabla$ wangshousen900@yeah.net)

Fujian Medical University https://orcid.org/0000-0001-9626-8586

\section{Xiangrong Chen}

Second Affiliated Hospital of Fujian Medical University

\section{Research}

Keywords: Cerebral venous circulation, Traumatic brain injury, Neuroinflammation, solTNF-a /NF-kB pathway

Posted Date: May 11th, 2021

DOl: https://doi.org/10.21203/rs.3.rs-487715/v1

License: (9) (1) This work is licensed under a Creative Commons Attribution 4.0 International License. Read Full License 


\section{Abstract}

Background: Traumatic brain injury (TBI) is recognized as a common cause of death and disability worldwide. Cerebrovascular reaction, as an important cause of secondary injury, can cause secondary bleeding, venous sinus thrombosis, and malignant brain swelling. Intracranial venous return disorder is closely related to patient prognosis. Treatment strategies after craniocerebral injury have primarily focused on the recanalization of cerebral arteries and capillaries and the protection of insulted neurons. In this study, we used an acute subdural hematoma (ASDH) model with cranial venous outflow obstruction (CVO) to explore the mechanism by which CVO aggravates the pathological process after TBI, especially for inflammation and tissue damage.

Methods: Based on a rat ASDH model using the modified Miller method, an intracranial venous circulation obstruction intervention was given to form a compound model. A sham operation group (sham), a CVO group, an ASDH group and an ASDH+CVO group were constructed. A neurobehavioural assessment and the determination of brain water content were performed. The content was determined by Evans blue staining. Expression levels of inflammatory factors and metalloproteinases ADAM17 and MMP9 were detected by immunofluorescence assay, ELISA and Western blot. The rats in the ASDH+CVO group were treated with XPro1595 to compare the changes in the NF-KB/MMP9 pathway before and after the inhibition of solTNF.

Results: CVO aggravated neurological function defects and damage to the blood-brain barrier in ASDH rats. CVO significantly increased the expression level of ADAM17, and localization of ADAM17 in intracranial cells occurred mainly in microglia or macrophages (positive for IBA-1), accompanied by increased secretion of solTNF. Activation of the ADAM17/solTNF/NF-KB pathway increased the subsequent inflammatory response and tissue damage.

Conclusions: The results of this study suggest that intracranial venous return disorder after TBI significantly promotes the expression of ADAM17, which is mainly located in microglia or macrophages, and that the secretion of solTNF- $\alpha$ is increased. The ADAM17/solTNF- $\alpha / N F-k B$ pathway plays an important role in the subsequent inflammatory response and tissue injury. Our study reveals a molecular basis for the finding that CVO aggravates inflammation and tissue damage.

\section{Background}

Traumatic brain injury (TBI) is recognized as a common cause of death and disability worldwide. Such injury can involve acute subdural hematoma, subarachnoid hemorrhage, contusion and laceration and other forms, with high mortality and disability rates[1]. The cerebrovascular reaction, as an important cause of secondary injury, can cause secondary bleeding, venous sinus thrombosis, and malignant brain swelling[2,3]. Treatment strategies after craniocerebral injury primarily focus on the recanalization of cerebral arteries and capillaries and the protection of insulted neurons. In recent years, clinical studies 
have confirmed that intracranial venous return disorder is closely related to the prognosis of patients[4$6]$.

Cranial venous outflow obstruction (CVO) aggravates the damage of the blood-brain barrier (BBB) and posttraumatic tissue edema. An important link is the destruction of BBB, which consists of endothelial cells and tight junctions (TJs), the basement membrane, astrocytes, and peripheral cells. Previous studies have indicated the activation of matrix metalloproteinases (MMPs), and disruption of the BBB is induced by inflammatory signalling in a rat TBI model. Inflammatory cytokines, such as tumour necrosis factor-a (TNF-a), can impair BBB integrity[7]. Endothelial cells connected by TJs constitute an integral component of the BBB. TJs are comprised of Occluding, Claudin, ZO-1 and other junction adhesion molecules that maintain the structural and functional integrity of the brain endothelium[8, 9]. The neuroinflammation induced by CVO is closely related to the impairment of the integrity of the blood-brain barrier.

Our previous studies have shown that the inflammatory response of the central nervous system is characterized by the activation of microglia cells which are unique to the central nervous system[10]. The microglia are polarized, releasing a large number of inflammatory mediators, such as TNF-a, IL-1 $\beta$, and IL6 , and inducing a neuroinflammatory response. TNF-a is a multifunctional, proinflammatory cytokine associated with all phases of TBI, from injury to repair and inflammatory effects[11]. It exists in two major forms, transmembrane (tmTNF) and soluble (solTNF) protein, the latter released via proteolytic cleavage by Recombinant A Disintegrin And Metalloprotease 17 (ADAM17) [12]. TNF acts via TNF receptor 1 and TNF receptor 2; When solTNF interacts with receptors on the surface of the cell membrane, the receptors activates RIP, which eventually activates IKK kinase by phosphorylating and dissociating the IKB-a protein from the trimer. Subsequently, the NF-KB dimer is exposed to a nuclear localization sequence (NLS), and phosphorylated p65 rapidly enters the nucleus from the cytoplasm, where it binds to a specific sequence on nuclear DNA and promotes the transcription of related genes. The NF-KB pathway is associated with the expression of numerous cytokines and is involved in the regulation of the inflammatory response, apoptosis and other pathophysiological processes through direct or indirect intercellular signalling pathway interactions $[13,14]$.

During the course of clinical treatment of $\mathrm{TBI}$, once the intracranial venous return is blocked, such as hypercoagulable state, venous sinus thrombosis, or damage to important intracerebral veins during surgery, the condition will worsen rapidly[15-17]. However, its specific molecular mechanism is still not very clear. There are certain limits to the conclusions that can be drawn using a model of only one type of TBI because head injury is often multifactorial. Exacerbating factors include not only primary brain damage but also secondary insults such as impairment of BBB integrity induced by obstruction of the cranial venous outflow. In this pathological process, neuroinflammation may play an important role. In the present study, we developed a new acute subdural hematoma (ASDH) model with CVO. The inflammatory factors and metalloproteinases ADAM17 and MMP9 were detected, and the potential molecular mechanisms focusing on ADAM17/solTNF-a/NF-KB pathway were also investigated.

\section{Materials And Methods}




\section{Animals}

Ethical approval for all experiments were granted by the 900th Hospital Ethics Committee (Fuzhou, China, 2020/50) and conducted under strict supervision. Adult male Sprague-Dawley rats, ranging between 280 and $320 \mathrm{~g}$, were purchased from the Animal Experimental Center of 900th Hospital. All animals were housed at room humidity $>50 \%$ and temperature $\left(24-26^{\circ} \mathrm{C}\right)$ with free access to water and food.

\section{Experimental model and grouping}

All rats were randomized into several different groups as follows: a sham group, a CVO group, an ASDH group, and an ASDH+CVO group (CVO operating $6 \mathrm{~h}$ after establishment of the ASDH model). After modeling, the groups were then divided into three subgroups: a 1-day group $(n=24)$, a 3-day group $(n=6)$, and a 7-day group $(n=6)$. Six rats in each group were used for neurological evaluation and the measurement of brain water content; six rats in the one-day group were sacrificed for histological studies. The remaining rats were used for immunofluorescence detection and Western blot studies. Another 24 rats with the ASDH+CVO model were used for pharmacological treatment. The ASDH rat model was made using the modified Miller method[18, 19]. First, anaesthesia was induced using $4 \%$ isoflurane, and then maintained with $2 \%$ isoflurane delivered by a small-animal anaesthetic machine. Every rat was fixed in the stereotactic frame. A cranial hole $2 \mathrm{~mm}$ in diameter was drilled $4 \mathrm{~mm}$ to the right of the sagittal suture and $4 \mathrm{~mm}$ posterior to the coronal suture. Using an operating microscope, the dura was incised. Then, nonheparinized venous blood $(0.4 \mathrm{ml})$ was injected into the subdural space by a blunt Olive Tip 8gauge needle, the hole was sealed, and the scalp was sutured. The injection procedure lasted over 7 min. In the sham group, holes were drilled in the sham group, but nothing was injected. The CVO rat model was established by ligating the retro-orbital venous plexus and petrosquamosal fissure venous plexus where the intracranial venous sinus is interconnected supraorbitally with the branches of facial vein and the superficial temporal vein, and returns to the external jugular venous system[20]. To validate the model, we monitored cortical cerebral blood flow (CBF) using laser speckle imaging as described previously[21].

\section{Pharmacological treatment}

XPro1595(Xencor Inc., Monrovia, United States) was administered intravenously once, at a dose of $10 \mathrm{mg} / \mathrm{kg}, 30$ minutes after ASDH+CVO model $(n=12)$. Saline was used as the vehicle. another group rats were injected with simple saline as a control.

\section{Assessment of neurological injury}

Neurological injury was assessed using modified neurological severity scores (mNSSs), which consist of evaluating the motor, sensory, and reflex examinations of rats. Neurological injury was recorded when when the corresponding reflex was lost or a task was completed unsuccessfully. The mNSS test was graded on a scale of $0-18$, in which a score of 0 indicated normal performance and a total score of 18 points indicated severe neurological deficits, 1-6 indicated mild injury, 7-12 indicated mean-moderate 
injury, and 13-18 indicated severe injury. Neurological deficits were measured 1, 3, and 7 days after the experiment by investigators who were blinded to the group information.

\section{Measurement of brain water content}

Brain water content was measured using the wet weight-dry weight method. After assessment of neurological injury, the rats were sacrificed by giving a lethal overdose of anesthesia, and the brains were removed immediately. the cerebral cortex was isolated at the edge of the bone ( $2 \mathrm{~mm}$ around craniotomy; $200 \pm 20 \mathrm{mg}$ ), excess blood and cerebrospinal fluid were removed with the help of filter paper. A digital scale was used to measure wet weight; samples were then placed in an oven to dry for $24 \mathrm{~h}$ at $100^{\circ} \mathrm{C}$. Dry weight was measured again for each sample. Brain water content was calculated according to the formula: Brain water content $(\%)=$ (total wet weight of brain-dry weight of brain)/total wet weight of brain $\times 100 \%$.

\section{Evans blue staining of the brain}

Measuring the extravasation of Evans blue was used to investigate BBB permeability[22]. Evans blue was intravenously injected $2 \mathrm{~h}$ before the rats were sacrificed 1 day after modelling. Rats were transcardially perfused with PBS immediately after sacrifice. The brains were quickly removed and homogenized in a solution with $1 \mathrm{~mL} 50 \%$ trichloroacetic acid. Then, the samples were centrifuged. The absorption of the supernatant was calculated via a spectrophotometer at a wavelength of $620 \mathrm{~nm}$. The quantity of Evans blue was measured according to a standard curve and expressed as micrograms of Evans blue/g of brain tissue.

\section{Transmission electron microscopy}

Sodium pentobarbital (1\%) was intraperitoneally injected to anaesthetize the rats deeply one day after modelling. Cardiac perfusion was performed with $4 \%$ paraformaldehyde, and then the brain tissue was carefully separated and fixed in a combination of 1.5\% paraformaldehyde and $3 \%$ glutaraldehyde for 24 $\mathrm{h}$, then followed by $1 \%$ osmic acid for $2 \mathrm{~h}$. The samples were embedded conventionally and sliced for uranyl acetate and lead citrate staining. Then, the sections were observed by a transmission electron microscope. The vascular lumen and endothelial cells were localized at a low magnification, and TJs were observed at a high magnification.

\section{Immunofluorescence analysis}

Cortical tissues under the compress of subdural hematoma were embedded in paraffin after formaldehyde-fixed and cut into $4 \mu \mathrm{m}$ thick sections. Slices went through xylene dewaxing, a graded series of alcohol, and a citric acid buffer protocol for antigen retrieval. For immunofluorescence, the sections were incubated overnight at $4{ }^{\circ} \mathrm{C}$ with antibodies against ADAM17(1:200, Abcam, Cambridge, UK), ionized calcium-binding adapter molecule (Iba-1) (1:200; Santa Cruz, CA, USA), glial fibrillary acidic protein (GFAP) (1:200, Abcam). After washing, the sections were then incubated with secondary goat antirabbit IgG antibodies (Alexa Fluor 488, 1:200, Invitrogen, NY, USA) for $1 \mathrm{~h}$ at room temperature. The cell 
nuclei were stained with 4',6-diamidino-2-phenylindole (DAPI). The positive cells in five selected ROIs were calculated under a microscope at 400 magnification by a pathologist who was blinded to the experimental groups.

\section{Enzyme-linked immunosorbent assay (ELISA)}

Inflammatory factors of brain tissue were measured using ELISA kits for TNF- $a$, IL-1 $\beta$, IL-6, IL-10, and HMGB1 (Boster Biotech, Wuhan, China). According to the manufacturer's instructions, samples and standards were sequentially incubated with respective monoclonal antibodies, biotinylated anti-rat antibody, and then horseradish peroxidase. The detected OD values were transformed into a concentration value.

\section{Western blotting analysis}

Brain tissues were rapidly dissected in ice-cold phosphate-buffered saline, and western blotting was performed as described previously[23]. We extracted proteins with radioimmunoprecipitation assay (RIPA) lysis buffer. $30 \mu \mathrm{g}$ of the proteins were loaded on a gel and separated by sodium dodecyl sulfatepolyacrylamide gel electrophoresis, and then transferred to polyvinylidene difluoride membranes and probed with primary antibodies against ADAM17, MMP9(1:500, Abcam), ZO-1(40-2200, Thermo Fisher Scientific, USA), Occludin (33-1500, Thermo Fisher Scientific), Iba-1, phosphorylated (p)-IkB, and NF-KB p65 (1:200, Cell Signaling Technology), followed by incubation with appropriate horseradish peroxidaseconjugated IgG (1:5000, Abcam) secondary antibodies. Chemiluminescent signals were acquired using the Millipore ECL Western Blotting Detection System (Millipore, Billerica, MA, USA). Expression levels were normalized against $\beta$-actin (1:5000, Abcam).

\section{Statistical analysis}

The statistical analyses were processed via SPSS 20.0 statistical software (IBM SPSS, Inc., Chicago, USA). The quantitative data were presented as the mean \pm standard deviation. Different groups were compared by one-way analysis of variance (ANOVA), and Student-Newman-Keuls tests were used for post hoc multiple comparisons. Values with a $p<0.05$ were considered statistically significant.

\section{Results}

\section{CVO produces CBF alterations and exacerbates neurological deficits and brain edema in rats with ASDH}

The intracranial venous return was blocked by ligating the retro-orbital venous plexus and petrosquamosal fissure venous plexus in rats. To test for changes in CBF, laser speckle contrast imaging was used to monitor cerebral cortex blood flow at $24 \mathrm{~h}$ after CVO. The results showed that after cerebral venous return was blocked, the blood flow rate of the cerebral hemisphere cortical arteries, veins and capillaries slowed. After $24 \mathrm{~h}$, the CBF decreased significantly, and the blood flow velocity of the contralateral hemispheric venous sinus increased (Fig.1c). 
The modified neurological deficit scale score was used to evaluate the neurological function of the rats in each experimental group after modelling. The results showed that the mNSS score of the sham group had no significant changes in each time period after the modelling, and the neurological function was normal. The rats in the CVO-only group had mild neurobehavioural impairment and could basically complete the relevant test actions. Compared with the rats in the CVO-only group and the ASDH group, the scores of neurological deficits in the ASDH+CVO group increased significantly at 1, 3, and 7 days after injury $(P<0.01)$, showing that ASDH rats with intracranial venous return disorder had more obvious neurological dysfunction. The destruction of the BBB and the degree of brain edema indirectly reflect the degree of neurological damage. Evans blue staining and brain water content measurements are often used to monitor BBB damage and the severity of cerebral edema. We used the above experiments to measure the damage in each experimental group. The results showed that after 1 day of intervention, the penetration and content of Evans blue in the brain tissue of the injured cerebral cortex significantly increased $(20.3 \pm 1.52$ VS $5.82 \pm 1.23,2.25 \pm 0.86, p<0.01)$ (Fig.1b). The water content of the cerebral hemisphere brain tissue of ASDH rats increased significantly on the first 1, 3, and 7 days after CVO intervention (Fig.1e).

\section{CVO aggravates the destruction of endothelial cell TJ proteins in rats with ASDH}

TJs between endothelial cells are important parts of the BBB, and a decrease in TJ proteins increases the permeability of the BBB. We observed the BBB structure of the cerebral cortex in the experimental group by using transmission electron microscopy. Compared to the sham group and the ASDH group, TJs between the endothelial cells in the ASDH+CVO group were sparser, suggesting that CVO intervention caused more damage to TJs (Fig.2a).

Western blot analyses were used to monitor the important TJ proteins ZO-1 and Occludin. The results revealed that CVO resulted in the downregulation of ZO-1 and Occludin in the cortex 1 day after ASDH. Compared to the ASDH group, the content of ZO- 1 decreased in the ASDH+CVO group $(25.32 \pm 7.45 \%$ VS. $32.74 \pm 4.95 \%, p<0.05)$, as well as did the content of Occludin( $42.38 \pm 3.82 \%$ VS. $60.55 \pm 4.68 \%, p<0.01)$ (Fig.2b-c). The metalloprotease MMP9, which is related to the destruction of the BBB, and ADAM17, which is related to the inflammatory reaction, were also detected. However, the results showed that both of these metalloproteases were significantly increased in the ASDH+CVO group compared with the ASDH group $(p<0.05)($ Fig.2c-d).

\section{Promoting the expression of inflammatory factors in ASDH rats after CVO intervention}

ELISA was used to detect the expression of inflammatory factors on the first day after modelling in each group. Compared to the sham group, the inflammatory factors TNF-a, IL-1 $\beta$, IL-6 and HMGB1 in the brain tissue of the ASDH and ASDH+CVO groups were significantly increased on day 1 , the expression of inflammatory factors was higher in the ASDH + CVO group than in the ASDH group $(p<0.05)$, and the anti-inflammatory factor IL-10 decreased in the ASDH and ASDH+CVO groups compared with the sham group(Fig.3a). Western blot results showed that compared to the sham and CVO-only groups, the ASDH and ASDH+CVO groups showed significantly increased expression of Iba-1 protein, which is a marker of 
microglia, and there was a significant increase in Iba- 1 in the ASDH+CVO group compared with the ASDH group ( $1.55 \pm 0.12$ VS $1.2 \pm 0.1, p<0.05$ ), while the astrocyte marker GFAP showed no significant changes in expression. The results suggested that CVO increased the activation of microglia on the basis of ASDH damage (Fig.3b).

\section{CVO promotes the activation of microglia and increases ADAM17 expression in microglia}

ADAM17 has been shown to hydrolyse TNF on the cell membrane and translate tmTNF to solTNF, which participates in the release of various inflammatory factors. Using double immunofluorescent staining, ADAM17 expression in microglia (Iba-1+) and astrocytes (GFAP+) was assessed. The results showed that ADAM17 was rarely expressed in the cortical tissue of the sham group. The expression levels of ADAM17 were higher in the ASDH group and ASDH+CVO group than in the sham group, and the expression of ADAM17 was significantly increased after CVO intervention. Moreover, ADAM17 was mainly expressed in microglia but not in astrocytes according to double immunofluorescent staining (Fig.4). The results suggested that CVO plays an important role in aggravating damage from TBI via microglial activation and ADAM17 overexpression, which may cause inflammatory cytokine release.

\section{Specific inhibition of solTNF-a supplementation inhibits the TNFR/NF-KB signalling pathway caused by CVO intervention}

As an important inflammatory factor, solTNF- $a$ is shed and secreted outside the cell by binding to the specific receptor TNFR, activating the intracellular TNF-a receptor-associated death domain protein TRAF2 and RIP (receptor-interacting protein) to cause a series of cascade reactions. Then, TRAF2 or RIP activates the NF-KB pathway by promoting NF-KB translocation and produces a large number of inflammatory cytokines and acute reactor proteins, such as MMP9. The results of Western blot analyses showed that compared with the corresponding levels in the ASDH group, the expression of TNFR1 and downstream RIP protein in the cerebral cortex increased significantly after CVO intervention for 1 day, and the phosphorylation ratios of IKB-a and NF-KB p65 increased (Fig.5A). XPro1595, a specific inhibitor of solTNF- $a$, reversed these changes. Immunofluorescence analyses also indicated that intravenous injection of XPro1595 could significantly inhibit NF-KB p65 translocation into the nucleus of cells, compared with that in the ASDH+CVO control group(Fig.5B).

\section{XPro1595 supplementationinfluences the expression of MMP9 in lesioned cortices of ASDH+CVO rats}

XPro1595, as a TNF-a synthesis inhibitor, can specifically bind to solTNF-a and block the effects of TNFa by preventing solTNF-a from binding to TNFR[24, 25]. Our Western blot and immunofluorescence experiments further detected the expression of MMP9 in the cerebral cortex of ASDH+CVO rats after XPro1595 intervention. Western blot results indicated that MMP9 expression in the ASDH+CVO group intravenously administered XPro1595 was significantly reduced compared with that in the control group (intravenous normal saline) $(p<0.01)$ (Fig. 6A). The results of immunofluorescence experiments also showed that XPro1595 supplementation decreased the fluorescence expression of MMP9 in the cortical injury area of the ASDH+CVO group (Fig.6B). 


\section{Discussion}

Vein drainage plays an important role in the pathophysiology of acute brain injury[4]. In our study, we conducted CVO intervention on the basis of ASDH rats to detect neurological dysfunction and BBB damage, and the results showed that after CVO intervention, the expression of inflammatory factors such as TNF- $\alpha, I L-1 \beta$, and IL- 6 in the cerebral cortex was significantly increased. Furthermore, intracranial venous return disorder after TBI significantly promotes the expression of ADAM17, which is mainly located in microglia or macrophages, and that the secretion of solTNF-a is increased. The ADAM17/solTNF-a/NF-KB pathway plays an important role in the subsequent inflammatory response and tissue injury.

Patients with only an intracranial venous circulation disorder have the most chronic or subacute onset in the clinic. However, compared with patients with venous sinus thrombosis alone, patients with secondary venous sinus thrombosis after trauma worsen rapidly with more severe clinical symptoms and have a higher risk of epilepsy, disturbance of consciousness and focal neurological function deficits[26, 27]. Atkinson et al.[28] simulated the rat model of chronic venous hypertension by ligating bilateral external jugular veins, and the results showed that compared with the sham operation group, the model group showed no significant differences in permeability, neuroinflammation, demyelination or clinical signs. In our study, there were no significant differences between CVO model rats and sham rats in neurological function damage, inflammatory response and BBB TJ proteins, which is consistent with previous research reports. However, in ASDH rats with a certain degree of damage to the BBB, intracranial venous return disorder will aggravate the inflammatory response and tissue edema. It is closely related to the high expression of ADAM17, solTNF-a and MMP9 in rats in the ASDH + CVO group.

TNF-a is considered as a multifunctional, proinflammatory cytokine associated with all pathological processes after TBI [11]. solTNF- $\alpha$ is expressed by different subsets of microglia and macrophages. solTNF-a is usually decomposed by ADAM17 and secreted to the outside of the cell and further activates downstream pathways by binding to the receptor $[29,30]$. When solTNF interacts with receptors on the surface of the cell membrane, the receptors activate solTNF-a/NF-KB pathway and promote the transcription of related inflammatory genes[31,32]. Previous studies also showed that ADAM17 can induce inflammation by regulating TNF-a-mediated expression of MMP9 in lung epithelial cells and prostate cancer patients[33,34]. ADAM17 is also closely related to the survival of oligodendrocytes and microglia in acute brain damage[35]. In the current study, we found that the normal cerebral cortex hardly expressed ADAM17, while the expression of ADAM17 in rats in the ASDH + CVO group was highly increased and located mainly in microglial cells or macrophages rather than astrocytes. The high expression of ADAM17 in microglial cells suggests that it is closely related to the inflammatory response after injury. we further tested the expression of the inflammatory pathway NF-KB in the experiment. The results of this study showed that in the ASDH rat model after CVO intervention, with the increase in solTNF-a, phosphorylated IкB- $a$ and NF-кB p65 were increased with the nuclear shift of p65. Moreover, the expression of MMP9 was increased, suggesting that there was a correlation between the expression of MMP9 and the solTNF-a/NF-kB pathway after CVO intervention. This relationship may be due to the 
further polarization of microglia in the central system after CVO intervention, the release of a large number of solTNF-a, and the positive feedback activation of the NF-KB pathway. It may also be that the BBB is damaged to a certain extent on the basis of ASDH. Under CVO intervention, CBF slows down, and blood pools in capillaries. Peripheral immune cells such as neutrophils and macrophages in the blood infiltrate into the interstitial brain tissue through the injured BBB and lead to the release of a large number of inflammatory factors.

Madsen et al.[36] used gene editing to achieve solTNF-a gene ablation and preservation of tmTNF in mice and found that the infarct volume of mice with permanent middle cerebral artery occlusion was reduced. Inhibition of TNF-a production or a reduction in TNF-a activity has become an effective anti-inflammatory treatment[37-40]. XPro1595 represents a new generation of TNF inhibitors that selectively neutralize the function of soluble TNF without affecting tmTNF or TNF receptors. XPro1595 is now used in clinical trials and scientific research[41-43]. To further study the mechanism by which CVO aggravates damage to rats with ASDH, we used XPro1595 to inhibit solTNF-a and found that it could reverse NF-KB pathway activation and reduce the expression of MMP9, which can destroy TJs between endothelial cells. These results indicate that The ADAM17/solTNF-a/NF-KB pathway plays an important role in the subsequent inflammatory response and tissue injury in the ASDH rat model after CVO intervention. Future studies involving ADAM17 knockout mice are warranted to further investigate the mechanisms underlying CVO intervention. Meanwhile, additional in vitro experiments are also needed to confirm the direct effects of CVO intervention on tissue damage.

\section{Conclusions}

In summary, intracranial venous return disorder after TBI promotes the expression of ADAM17 significantly, and the intracranial cells were mainly located in microglia or macrophages, and the secretion of solTNF-a was increased. ADAM17/solTNF- $a$ /NF-KB pathway plays an important role in subsequent inflammatory response and tissue injury. Our study reveals a molecular basis for the finding that CVO aggravates inflammation and tissue damage. Treatments focusing on ADAM17/solTNF- $a$ /NF$\mathrm{KB}$ pathway may provide a new strategy for the patients of TBI with blood circulation disorders.

\section{Abbreviations}

BBB: Blood-brain barrier; CVO: Cranial venous-outflow obstruction; ADAM17: A Disintegrin And Metalloprotease 17; MMP9: Matrix metalloproteinase 9; Iba-1: lonized calcium bindingadaptor molecule1; GFAP: Glial fibrillary acid protein; TBI: Traumatic brain injury; ASDH: acute subdural hematoma; HMGB1: High-mobility group box 1; TNF: Tumor necrosis factor; IL: Interleukin; mNSS: NF- KB: Nuclear factor-KB; TRAD: TNF receptor-associated death domain; NLS: Nuclear localization sequence

\section{Declarations}

Acknowledgements 
We would like to thank Dr. Heping Zheng, Department of Central Laboratory, the 900th Hospital Hospital, for advice and expert technical support. Sincere appreciation is also given to the teachers from department of Neurosurgery of 900th Hospital. We further thank Rong Chen for her support in routine histological procedures.

\section{Funding}

This work was financially supported by Science and Technology Innovation Joint Fund Project of Fujian Province, China (2019Y9045) and Fujian Province Scientific Foundation (2019J01168).

\section{Availability of data and materials}

The data supporting the conclusions of this article are included within the article.

\section{Authors' contributions}

CW contributed to the conception and design and writing of the manuscript. LX, SZ, ZZ and JL supported several experiments and helped in the acquisition of data and analysis and interpretation of data. YF and $\mathrm{KL}$ helped in the statistical analysis and revision of the manuscript. SW and XC helped in the technical support, obtaining of funding, conception and design, and revision of the manuscript. All authors read and approved the final manuscript.

\section{Ethics approval and consent to participate}

The experimental protocols in the present study including all the surgical procedures and animal usages conformed to the guidelines for the care and use of laboratory animals by the National Institutes of Health $(\mathrm{NIH})$ and were approved by the 900th Hospital Ethics Committee (Fuzhou, China, 2020/50).

\section{Consent for publication}

All authors have been consented for publication of the manuscript.

\section{Competing interests}

The authors declare that they have no competing interests.

\section{Author details}

${ }^{1}$ Fuzong Clinical Medical College of Fujian Medical University, Fuzhou 350025, Fujian, China

${ }^{2}$ Department of Neurosurgery, The First Affiliated Hospital of Wannan Medical College, Wuhu 241000, Anhui, China

${ }^{3}$ Department of Neurosurgery, 900th Hospital, Fuzhou 350025, Fujian, China 
${ }^{4}$ Department of Neurosurgery, The Second Affiliated Hospital of Fujian Medical University, Quanzhou 362000, Fujian, China.

\section{References}

1. Capizzi A, Woo J, Verduzco-Gutierrez M. Traumatic Brain Injury: An Overview of Epidemiology, Pathophysiology, and Medical Management. Med Clin North Am. 2020;104(2):213-38.

2. Chen J, et al. Biological characteristics of the cerebral venous system and its hemodynamic response to intracranial hypertension. Chin Med J (Engl). 2012;125(7):1303-9.

3. Salehi A, Zhang JH, Obenaus A. Response of the cerebral vasculature following traumatic brain injury. J Cereb Blood Flow Metab. 2017;37(7):2320-39.

4. Stolz E, et al. Intracranial venous hemodynamics in patients with midline dislocation due to postischemic brain edema. Stroke. 2002;33(2):479-85.

5 . West $\mathrm{JL}$, et al. Correlation between angiographic stenosis and physiologic venous sinus outflow obstruction in idiopathic intracranial hypertension. J Neurointerv Surg. 2019;11(1):90-4.

6. Liu M, et al. Patterns of chronic venous insufficiency in the dural sinuses and extracranial draining veins and their relationship with white matter hyperintensities for patients with Parkinson's disease. J Vasc Surg. 2015;61(6):1511-20.e1.

7. Rochfort KD, Cummins PM. The blood-brain barrier endothelium: a target for pro-inflammatory cytokines. Biochem Soc Trans. 2015;43(4):702-6.

8. Liebner S, et al. Functional morphology of the blood-brain barrier in health and disease. Acta Neuropathol. 2018;135(3):311-36.

9. Obermeier B, Daneman R, Ransohoff RM. Development, maintenance and disruption of the bloodbrain barrier. Nat Med. 2013;19(12):1584-96.

10. Chen $X$, et al. Omega-3 polyunsaturated fatty acid attenuates the inflammatory response by modulating microglia polarization through SIRT1-mediated deacetylation of the HMGB1/NF-KB pathway following experimental traumatic brain injury. J Neuroinflammation. 2018;15(1):116.

11. Shao X, et al. TNF-a-induced p53 activation induces apoptosis in neurological injury. J Cell Mol Med. 2020;24(12):6796-803.

12. Moss ML, Minond D, Recent Advances in ADAM17 Research: A Promising Target for Cancer and Inflammation. Mediators Inflamm, 2017. 2017: 9673537.

13. Dresselhaus EC, Meffert MK. Cellular Specificity of NF-KB Function in the Nervous System. Front Immunol. 2019;10:1043.

14. Shih RH, Wang CY, Yang CM. NF-kappaB Signaling Pathways in Neurological Inflammation: A Mini Review. Front Mol Neurosci. 2015;8:77.

15. Zhang J, Zhang F, Dong JF. Coagulopathy induced by traumatic brain injury: systemic manifestation of a localized injury. Blood. 2018;131(18):2001-6. 
16. Parker SL, et al. Management of Venous Sinus-Related Epidural Hematomas. World Neurosurg. 2020;138:e241-50.

17. Phelan HA. Pharmacologic venous thromboembolism prophylaxis after traumatic brain injury: a critical literature review. J Neurotrauma. 2012;29(10):1821-8.

18. Miller JD, et al. Ischemic brain damage in a model of acute subdural hematoma. Neurosurgery. 1990;27(3):433-9.

19. Xian L, et al. The construction of an improved model of acute subdural hematoma in rats. J Neurosci Methods. 2021;351:109075.

20. Mancini M, et al. Head and Neck Veins of the Mouse. A Magnetic Resonance, Micro Computed Tomography and High Frequency Color Doppler Ultrasound Study. PLoS One. 2015;10(6):e0129912.

21. Wang $C$, et al. Visualization of cortical cerebral blood flow dynamics during craniotomy in acute subdural hematoma using laser speckle imaging in a rat model. Brain Res. 2020;1742:146901.

22. Hopp $S$, et al. Alleviation of secondary brain injury, posttraumatic inflammation, and brain edema formation by inhibition of factor XIla. J Neuroinflammation. 2017;14(1):39.

23. Cheng $Y$, et al. Stress-induced neuroinflammation is mediated by GSK3-dependent TLR4 signaling that promotes susceptibility to depression-like behavior. Brain Behav Immun. 2016;53:207-22.

24. Yli-Karjanmaa M, et al. Topical Administration of a Soluble TNF Inhibitor Reduces Infarct Volume After Focal Cerebral Ischemia in Mice. Front Neurosci. 2019;13:781.

25. Zhou KX, et al. XPro1595 ameliorates bone cancer pain in rats via inhibiting p38-mediated glial cell activation and neuroinflammation in the spinal dorsal horn. Brain Res Bull. 2019;149:137-47.

26. Dobbs TD, et al. Cerebral venous sinus thrombosis complicating traumatic head injury. J Clin Neurosci. 2012;19(7):1058-9.

27. Chen $L$, et al. Insufficient cerebral venous drainage predicts early edema in acute intracerebral hemorrhage. Neurology. 2019;93(15):e1463-73.

28. Atkinson $\mathrm{W}$, et al. Ligation of the jugular veins does not result in brain inflammation or demyelination in mice. PLoS One. 2012;7(3):e33671.

29. Gilmore TD. Introduction to NF-kappaB: players, pathways, perspectives. Oncogene. 2006;25(51):6680-4.

30. Zhang Q, Lenardo MJ, Baltimore D. 30 Years of NF-KB: A Blossoming of Relevance to Human Pathobiology. Cell. 2017;168(1-2):37-57.

31. Clausen $\mathrm{BH}$, et al. Interleukin-1 beta and tumor necrosis factor-alpha are expressed by different subsets of microglia and macrophages after ischemic stroke in mice. J Neuroinflammation. 2008;5:46.

32. Tuttolomondo A, Pecoraro R, Pinto A. Studies of selective TNF inhibitors in the treatment of brain injury from stroke and trauma: a review of the evidence to date. Drug Des Devel Ther. 2014;8:222138. 
33. Li YQ, et al. ADAM17 mediates MMP9 expression in lung epithelial cells. PLoS One. 2013;8(1):e51701.

34. Xiao LJ, et al. ADAM17 targets MMP-2 and MMP-9 via EGFR-MEK-ERK pathway activation to promote prostate cancer cell invasion. Int J Oncol. 2012;40(5):1714-24.

35. Vidal PM, et al. ADAM17 is a survival factor for microglial cells in vitro and in vivo after spinal cord injury in mice. Cell Death Dis. 2013;4(12):e954.

36. Madsen PM, et al. Genetic ablation of soluble tumor necrosis factor with preservation of membrane tumor necrosis factor is associated with neuroprotection after focal cerebral ischemia. J Cereb Blood Flow Metab. 2016;36(9):1553-69.

37. Roy AK, Basir FA, Roy PK. A vivid cytokines interaction model on psoriasis with the effect of impulse biologic (TNF-a inhibitor) therapy. J Theor Biol. 2019;474:63-77.

38. Keane $\mathrm{J}$, et al. Tuberculosis associated with infliximab, a tumor necrosis factor alpha-neutralizing agent. N Engl J Med. 2001;345(15):1098-104.

39. Chaabo K, Kirkham B. Rheumatoid Arthritis - Anti-TNF. Int Immunopharmacol. 2015;27(2):180-4.

40. Deodhar A, Yu D. Switching tumor necrosis factor inhibitors in the treatment of axial spondyloarthritis. Semin Arthritis Rheum. 2017;47(3):343-50.

41. MacPherson KP, et al. Peripheral administration of the soluble TNF inhibitor XPro1595 modifies brain immune cell profiles, decreases beta-amyloid plaque load, and rescues impaired long-term potentiation in 5xFAD mice. Neurobiol Dis. 2017;102:81-95.

42. Eidson LN, et al. Chronic psychological stress during adolescence induces sex-dependent adulthood inflammation, increased adiposity, and abnormal behaviors that are ameliorated by selective inhibition of soluble tumor necrosis factor with XPro1595. Brain Behav Immun. 2019;81:305-16.

43. Barnum CJ, et al. Peripheral administration of the selective inhibitor of soluble tumor necrosis factor (TNF) XPro®1595 attenuates nigral cell loss and glial activation in 6-OHDA hemiparkinsonian rats. J Parkinsons Dis. 2014;4(3):349-60.

\section{Figures}




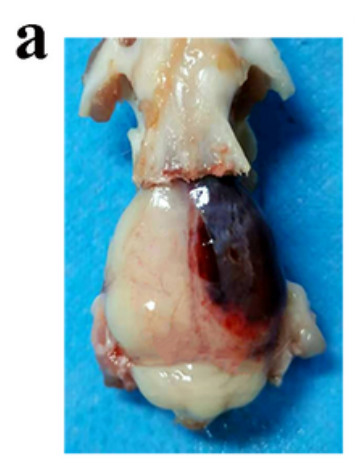

c

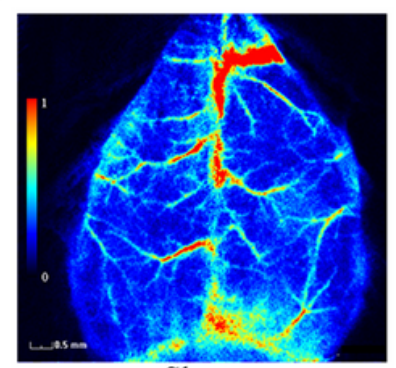

Sham b

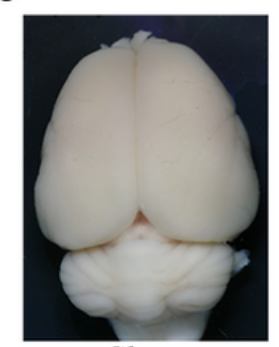

Sham

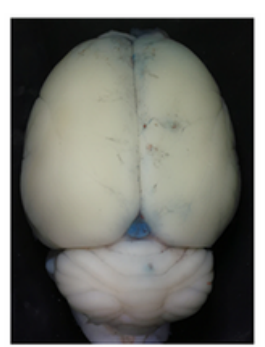

CVO

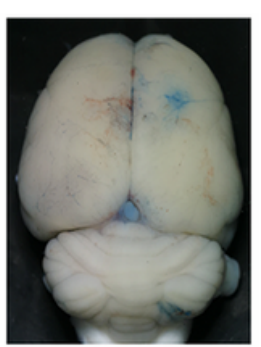

$\mathrm{ASDH}$
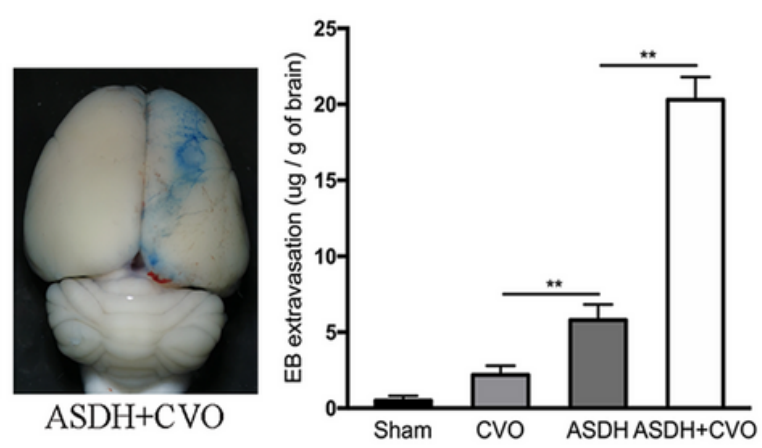

e

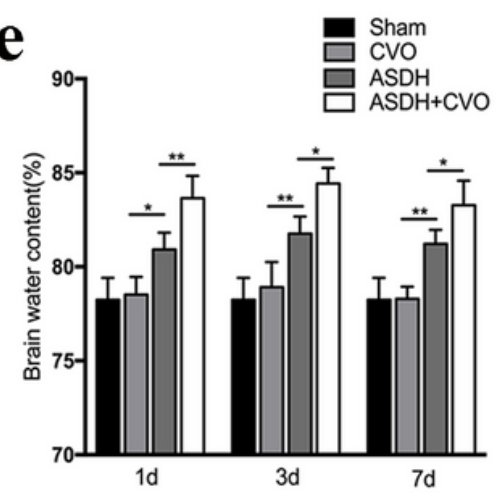

Figure 1

CVO increased neurological dysfunction, cerebral edema and BBB permeability after ASDH. a The acute subdural hematoma model in rats. The hematoma was located under the dura and compressed the surrounding brain tissue. $\mathrm{b}$ The impaired BBB function after CVO and a representative image of Evans blue dye extravasation in the experimental group. According to the Evans blue standard curve, the concentration of Evans blue in the specimen was measured using a microplate reader. Compared with the sham operation group and the unilateral $\mathrm{CVO}$ or ASDH group, the CVO+ASDH group had more Evans blue dye extravasation in the cortex at 1 day after modelling ( $20.3 \pm 1.52$ VS $5.82 \pm 1.23,2.25 \pm 0.86, p<0.01)$. c One day after CVO modelling, laser speckle imaging showed that venous blood flow of the ipsilateral cerebral hemisphere was blocked, and cerebral cortex blood flow decreased significantly. d CVO after ASDH aggravated the neurological deficit. Compared with that of the ASDH group, the mNss score of the CVO+ASDH group increased on days 1,3 , and 7 after modelling $(p<0.01)$. e The water content of the brain tissue increased; the bar graph shows the water content at 1 day, 3 days, and 7 days after modelling. Values are expressed as the mean \pm standard deviation $(n=6$ per group $),{ }^{*} p<0.05,{ }^{\star *} p<0.01$. 
$\mathbf{a}$
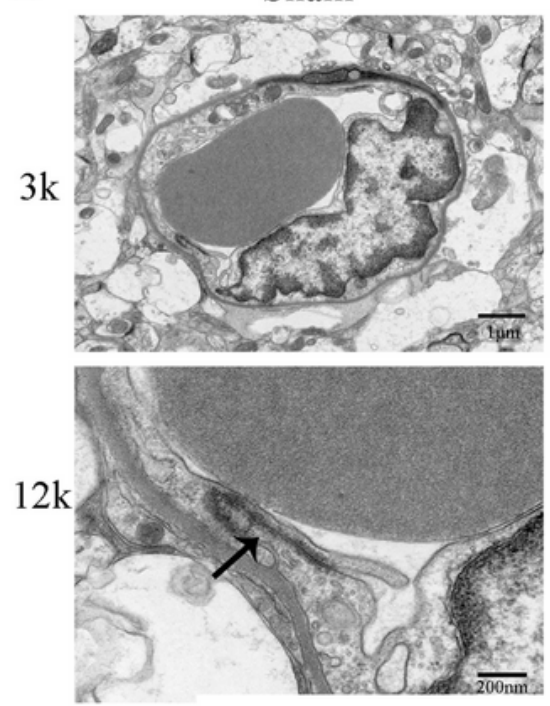

b
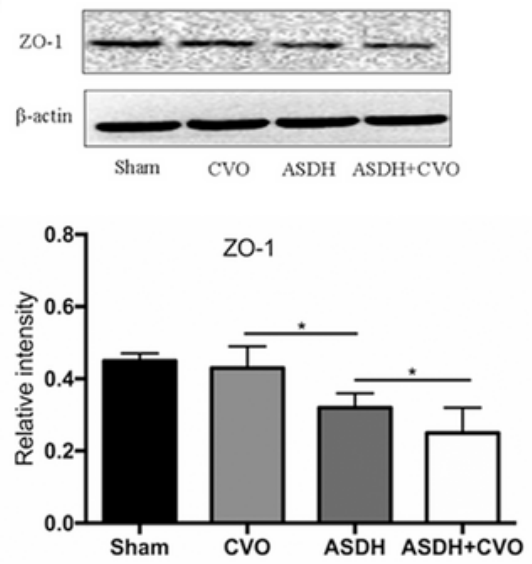

d
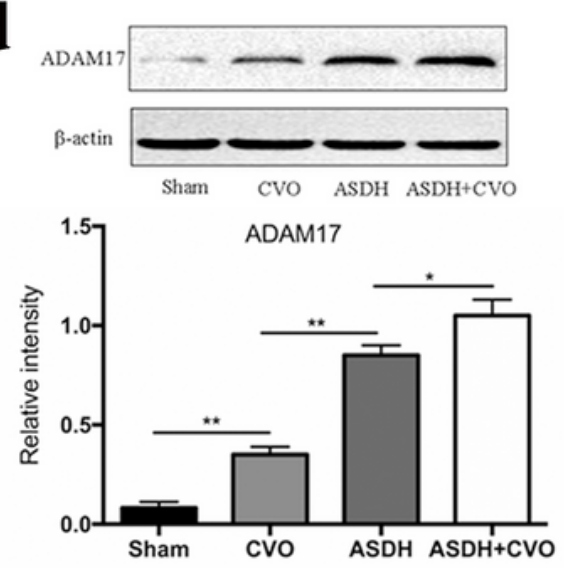

$\mathrm{ASDH}$
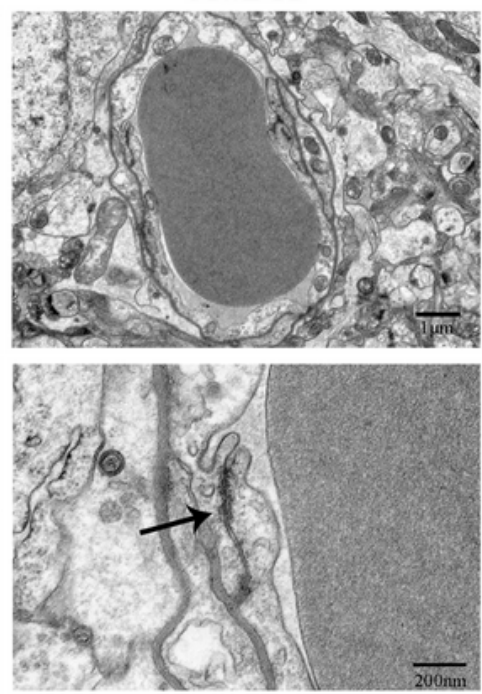

c
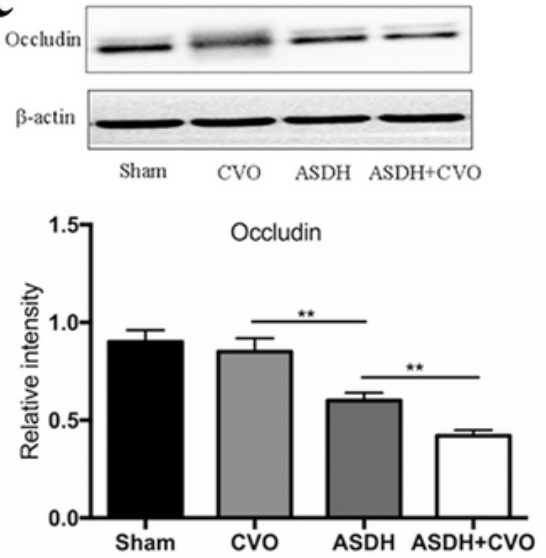

$\mathbf{e}$
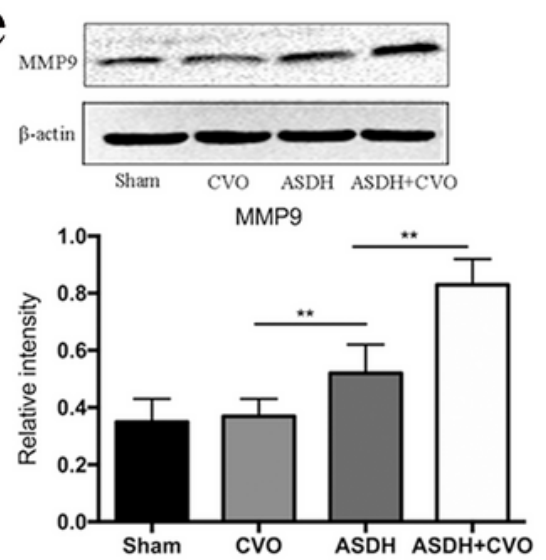

Figure 2

CVO aggravated the destruction of TJ proteins between endothelial cells of the BBB in ASDH rats. a Transmission electron microscopy showed the TJ structure as a black band between endothelial cells as the dense band shown by the black arrow. The magnified image shows the TJs between endothelial cells presented as integrated and continuous electron-dense bands (black arrow), which were sparse in the ASDH+CVO group. b-e Western blot results showed the changes in the content of TJ proteins ZO-1 and 
Occludin and metalloproteinases ADAM17 and MMP9. With the help of the system's software for quantitative analysis, compared with the ASDH group, in the ASDH+CVO group ZO-1 decreased $(p<0.05)$, the content of Occludin decreased $(p<0.01)$, the content of ADAM17 increased $(p<0.05)$, and the content of MMP-9 increased $(p<0.01)$, ${ }^{*}<0.05,{ }^{*} p<0.01$.

$\mathbf{a}$

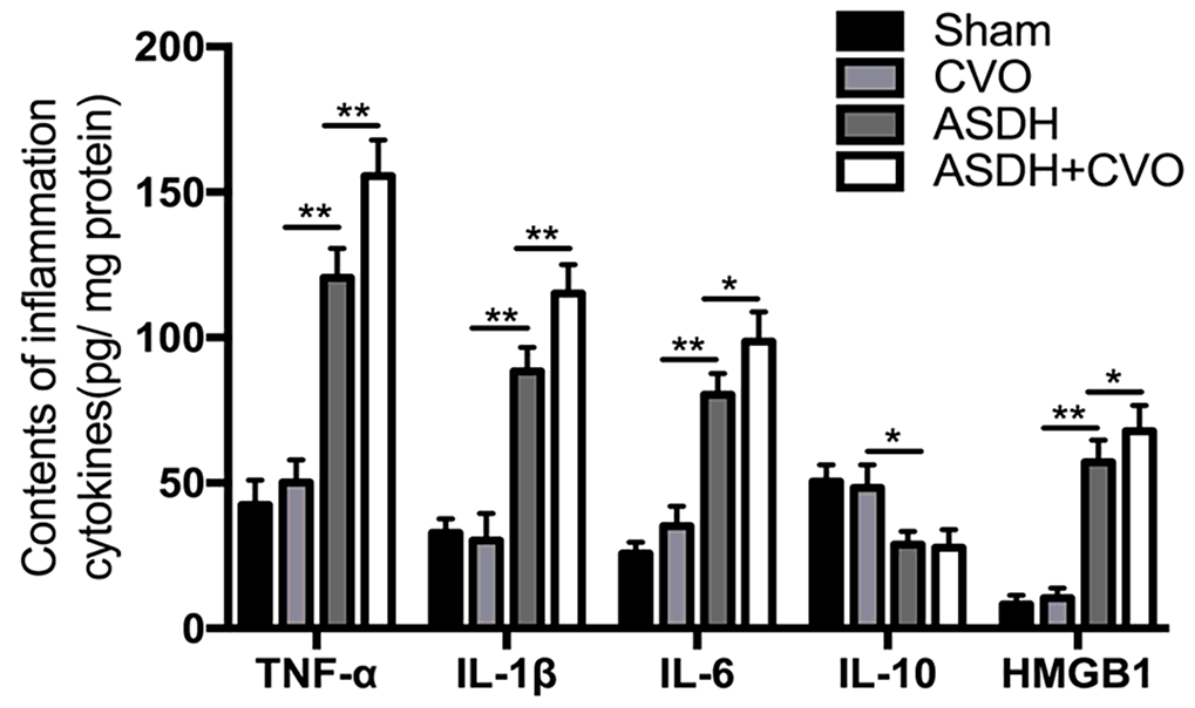

b
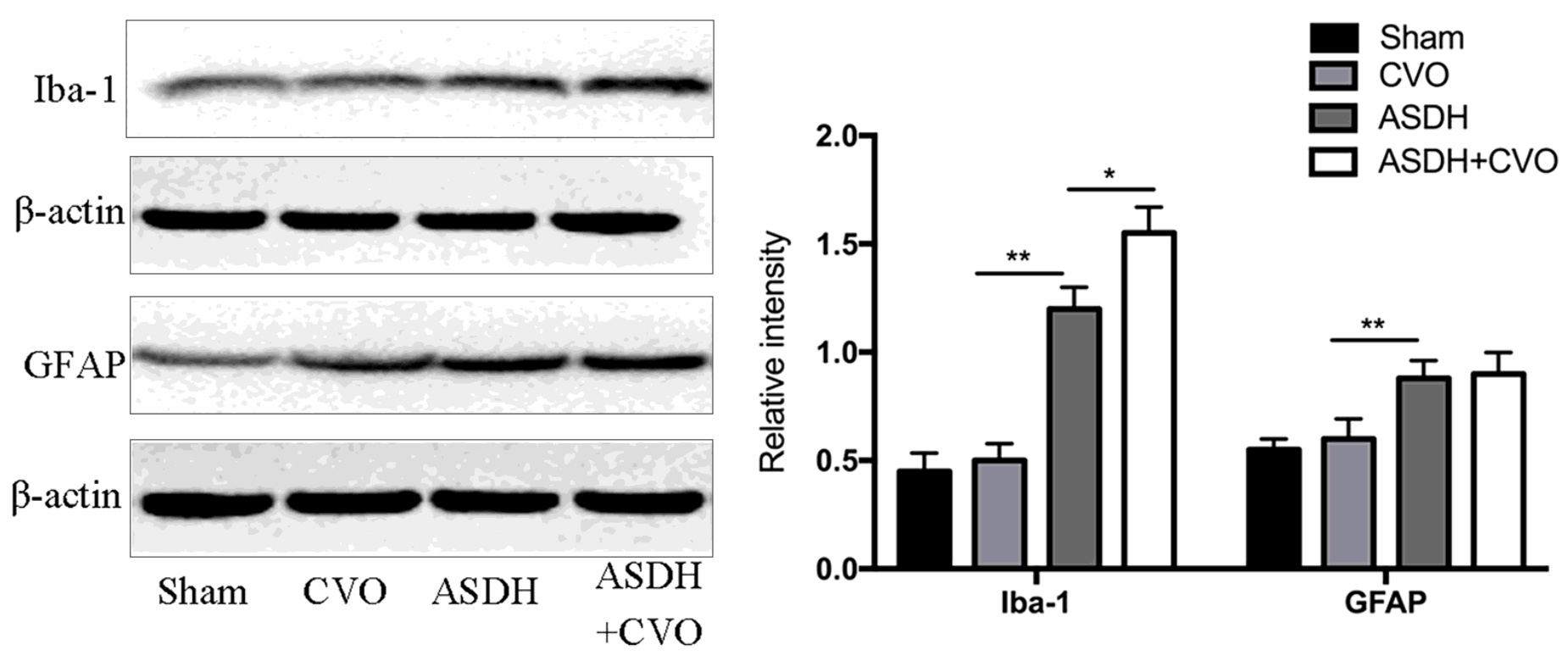

Figure 3

CVO increased microglial activation and the subsequent inflammatory response. a ELISA results showed that CVO increased the expression of TNF- $a, \mathrm{IL}-1 \beta, \mathrm{IL}-6$ and HMGB1 after ASDH injury while decreasing the expression of anti-inflammatory IL-10 factors $(p<0.05)$. b After cranial venous outflow was blocked, the microglial marker Iba- 1 increased significantly compared with that in the ASDH group $(1.55 \pm 0.12$ vs. $1.2 \pm 0.1, p<0.05$ ), while the astrocyte marker GFAP showed no significant changes in expression; $n=6$ in each group. The values are expressed as the mean \pm standard deviation, $* p<0.05,{ }^{\star} * p<0.01$. 


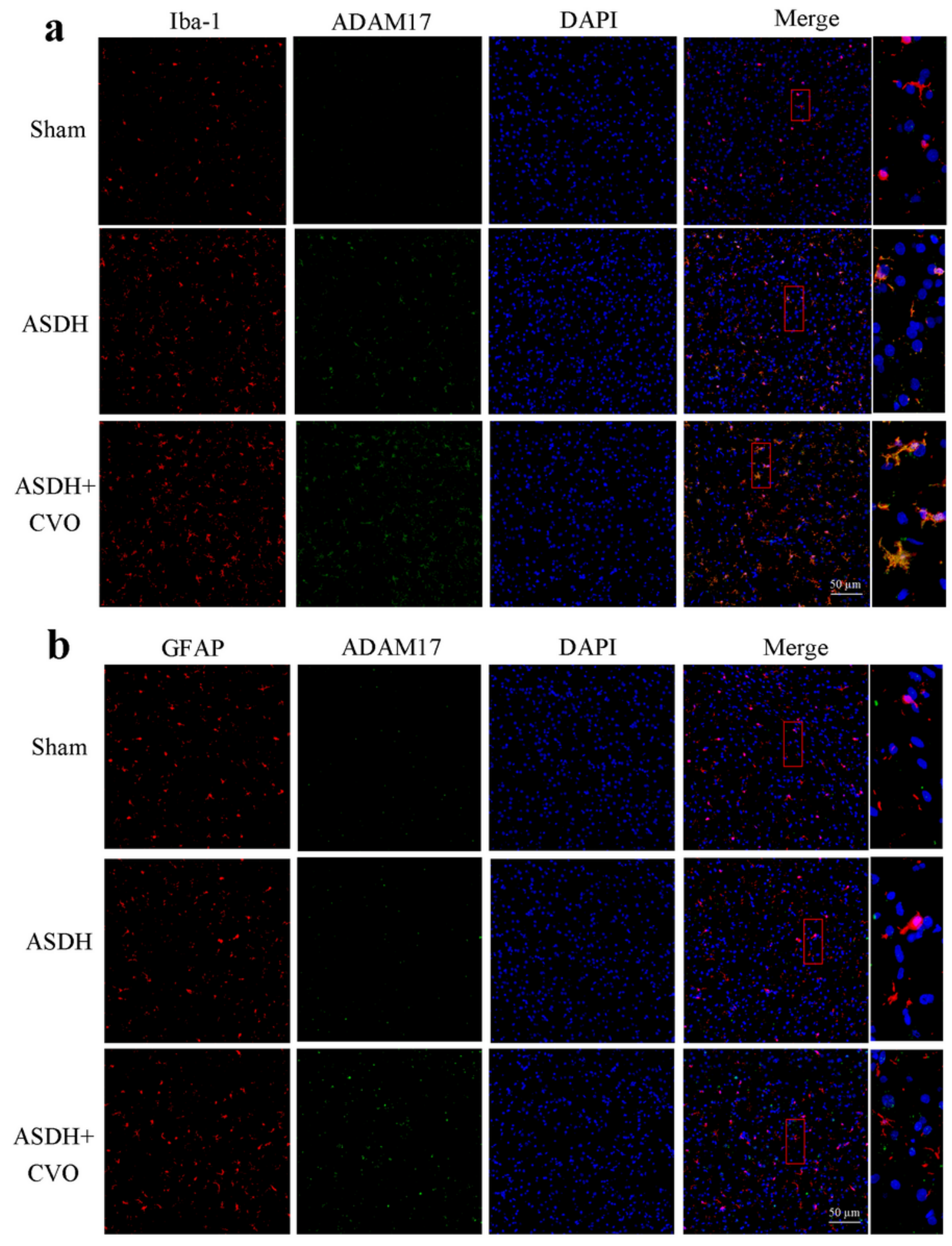

\section{Figure 4}

CVO increased metalloproteinase ADAM17 expression in lesioned cortices 1 day after modelling. Immunofluorescence double-labelling staining showed the expression of ADAM17 in microglia (Iba-1) and astrocytes (GFAP+) in the compressed cerebral cortex. a ASDH aggravated microglial activation, CVO further increased the microglial marker Iba-1, and the expression of ADAM17 in microglia significantly 
increased. b After ASDH and CVO modelling, the expression of the astrocyte marker GFAP did not change significantly, and ADAM17 in astrocytes in the ASDH+CVO group increased slightly. Scale bar=50 $\mu \mathrm{m}$.

a

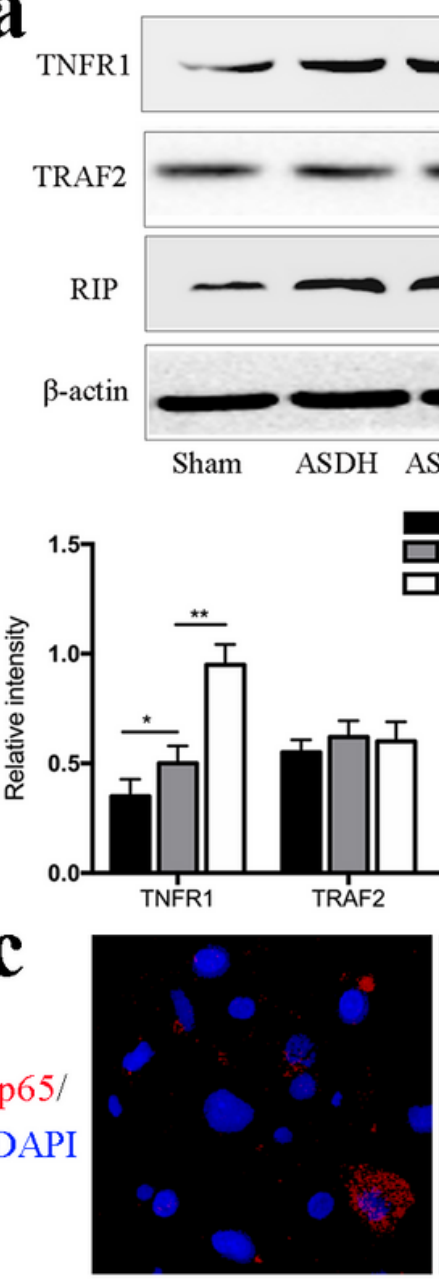

Sham b
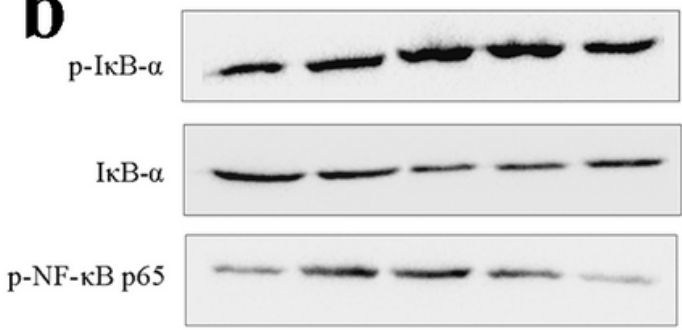

NF-кB p 65

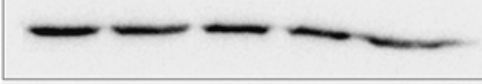

B-tubulin

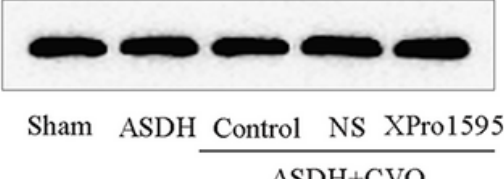

$\mathrm{ASDH}+\mathrm{CVO}$

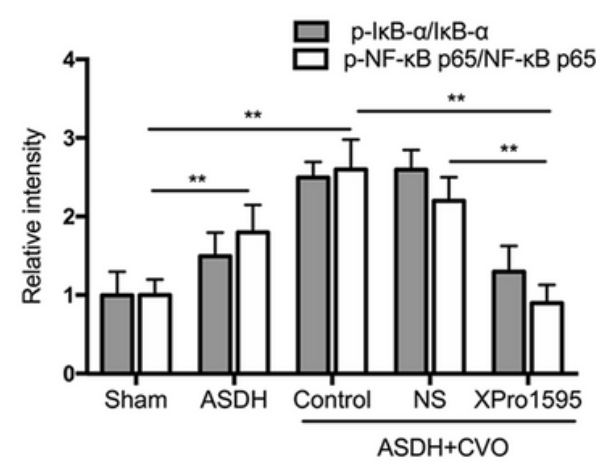

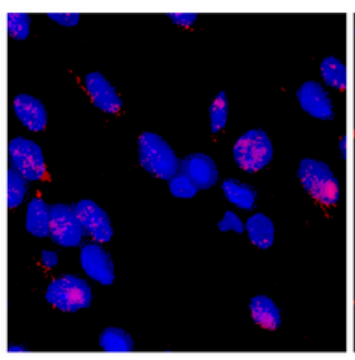

Control

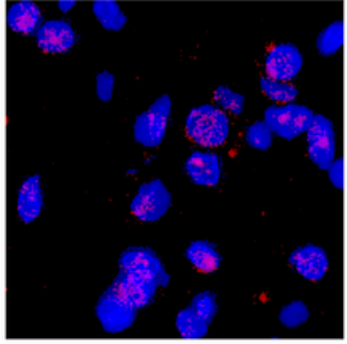

NS

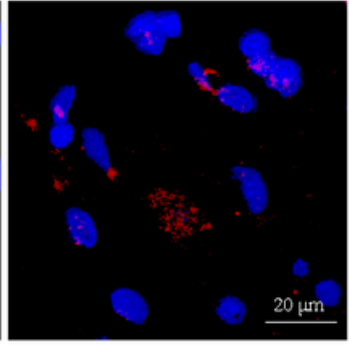

XPro1595

$\mathrm{ASDH}+\mathrm{CVO}$

\section{Figure 5}

CVO activated the TNF-a/NF-KB pathway in the cortex of ASDH rats one day after modelling. a Western blot analysis was used to detect TNFR1 and downstream pathway proteins in cortical tissues. The results showed that the expression of TNFR1 and downstream RIP protein increased in the cortical tissue cells of the ASDH+CVO group compared with the control group and ASDH group $(p<0.01)$, and the content of TRAF2 did not change significantly. b Western blot analysis showed representative results of IкB-a, p-IKBa, p-NF-кB p65 and NF-KB p65. The ASDH+CVO group was treated with intravenous injection of saline and the solTNF-a antagonist XPro1595 $(n=6)$. The measured values of each group of rats were standardized and semi-quantitatively analysed with $\beta$-tubulin protein, and the results are expressed as the ratios $p-I_{k B-a / l k B-a}$ and $p-N F-\kappa B$ p65/NF-kB p65. CVO increased these two ratios, and after XPro1595 intervention, the phosphorylation of IKB- $\alpha$ and NF-KB p65 was inhibited $(p<0.01)$. c Representative images of NF-KB p65 immunofluorescence staining in the experimental group, showing that p65 entered 
the nucleus, CVO promoted entry of p65 into the nucleus, and XPro1595 intervened to reverse this process. * $p<0.05$, ** $p<0.01$, scale bar $=20 \mu \mathrm{m}$.

\section{a}
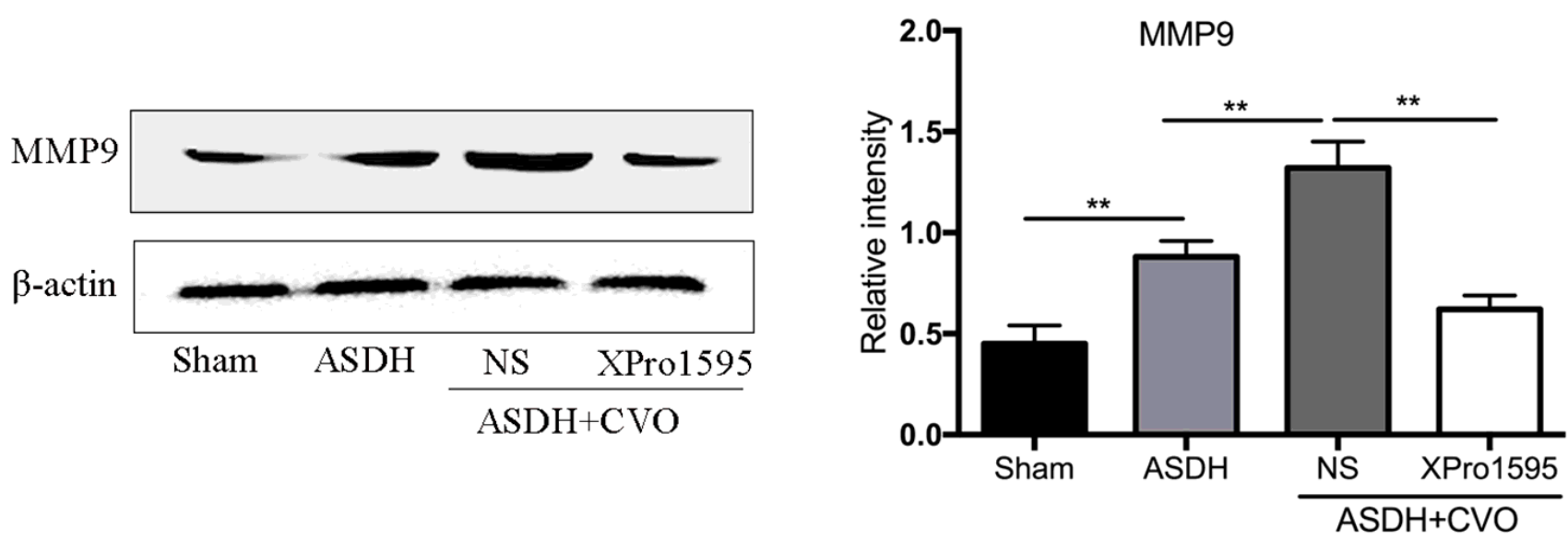

D

MMP9

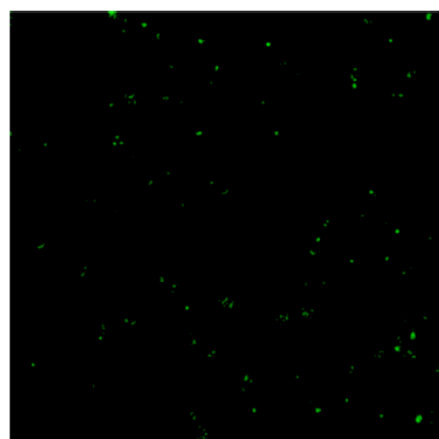

Sham

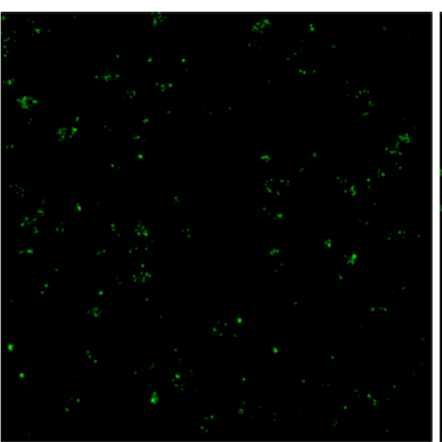

ASDH

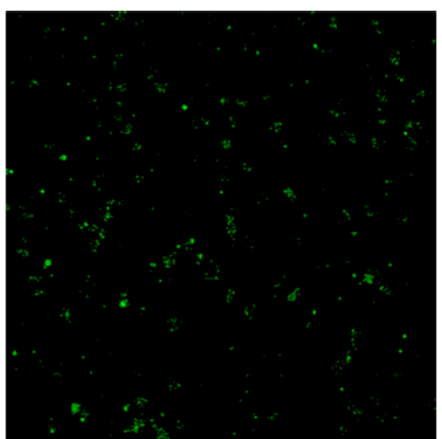

NS

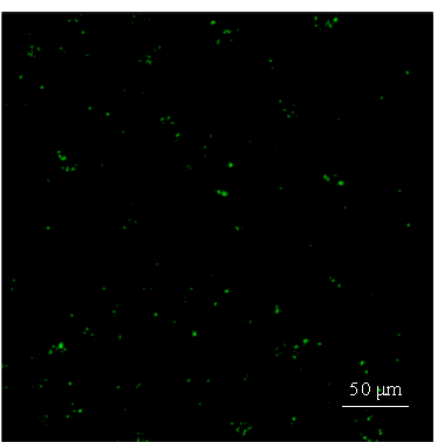

XPro1595

$\mathrm{ASDH}+\mathrm{CVO}$

\section{Figure 6}

The solTNF-a antagonist XPro1595 inhibited the expression of metalloproteinase MMP9 in ASDH rats with intracranial venous reflux disorder. a Western blot analysis showed that the expression of MMP9 in the ASDH+CVO group was lower than that in the control group and ASDH group after XPro1595 intervention ( $p$ <0.01). XPro1595 antagonized solTNF- $a$ and inhibited the activation of the solTNF-a/NFKB/MMP9 pathway, which was secondary to the CVO effect. b Immunofluorescence staining showed that XPro1595 inhibited the overexpression of MMP9 in the ASDH+CVO group. ** $p<0.01$, scale bar $=50 \mu \mathrm{m}$. 